SISTEMAS SOCIAIS COMPLEXOS

E INTEGRAÇÃO DE GEODADOS

NO DIREITO E NAS POLÍTICAS

ATAS DO COLÓQUIO

COORDENAÇÃO

Alexandra Aragáo

José Gomes dos Santos
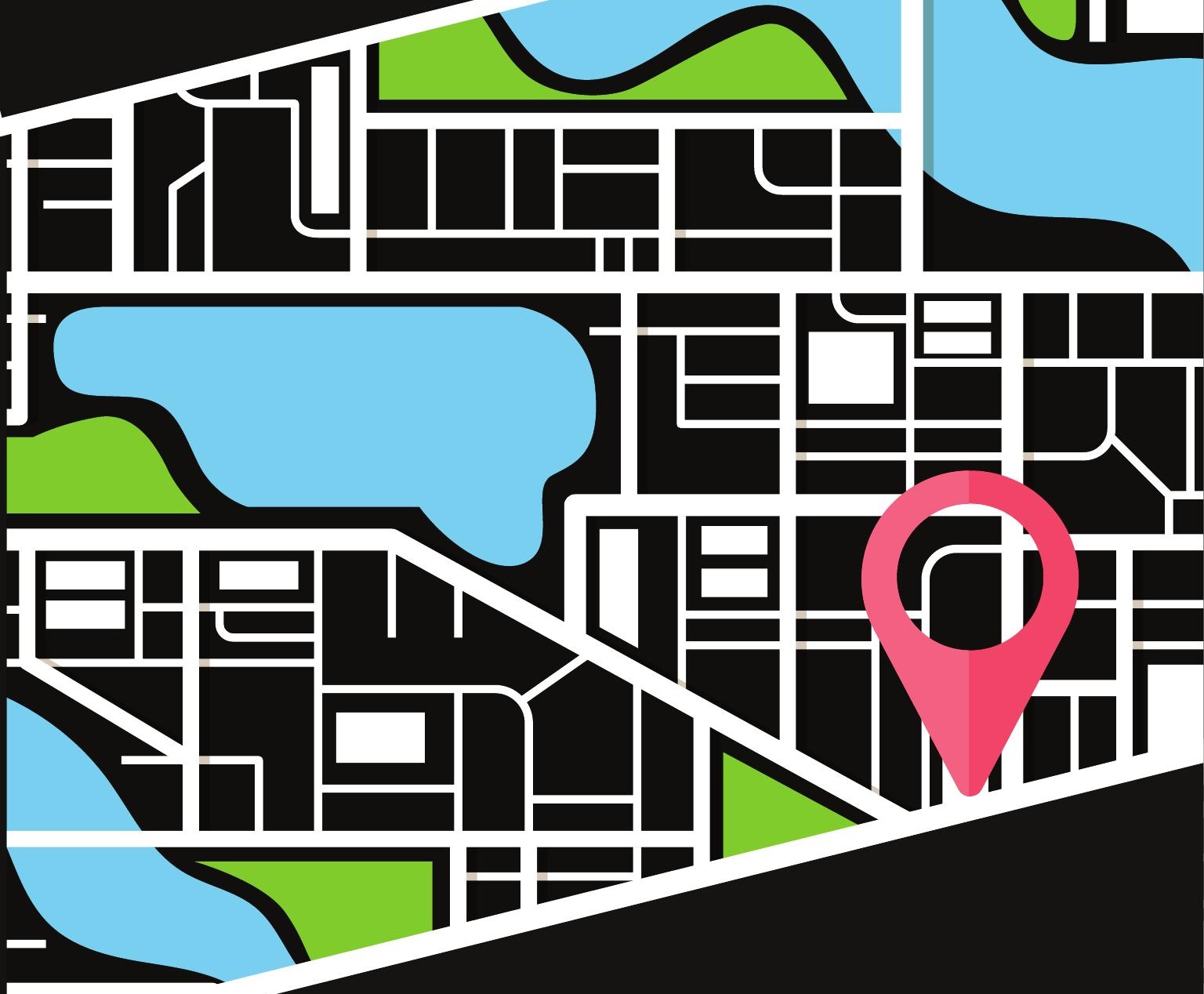

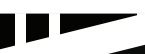

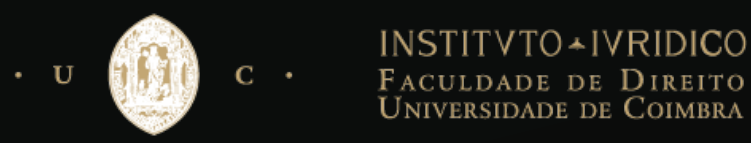




\title{
SISTEMAS SOCIAIS COMPLEXOS E INTEGRAÇÃO DE GEODADOS NO DIREITO E NAS POLÍTICAS
}

ATAS DO COLÓqUIO

\author{
COORDENAÇÃO \\ Alexandra Aragão \\ José Gomes dos Santos
}

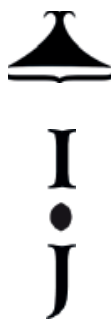


O presente livro foi realizado no âmbito da Rede temática "Just Side - Justiça e Sustentabilidade do Território através de Infraestruturas de Dados Espaciais", coordenada pelo Instituto Jurídico da Faculdade de Direito da Universidade de Coimbra e apoiada pelo programa CYTED-Ciencia y Tecnologia para el Desarrollo.

TÍTULO

Sistemas Sociais Complexos e Integração de Geodados no Direito e nas Políticas COORDENAÇÃO

Alexandra Aragão | José Gomes dos Santos

EDITOR

Instituto Jurídico da Faculdade de Direito da Universidade de Coimbra

ISBN

$978-989-8891-66-2$

CONCEPÇÃO GRÁFICA

Ana Paula Silva (capa) | Sara Baptista (miolo)

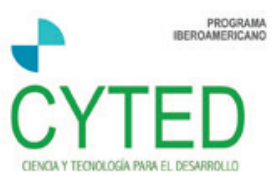




\section{Índice}

PREÂMBULO $\ldots \ldots \ldots \ldots \ldots \ldots \ldots \ldots \ldots \ldots \ldots \ldots \ldots \ldots \ldots \ldots \ldots$ vii

APRESENTAÇÃO .........................

\section{PARTE I - JUSTIÇA TERRITORIAL ATRAVÉS DA \\ CARTOGRAFIA. COMO E PARA QUÊ? ................}

- $01 \cdot$

O EMPREGO DE INFRAESTRUTURA DE DADOS ESPACIAIS PELO MINISTÉRIO PÚBLICO NO BRASIL PARA A CONCRETIZAÇÃO DOS VALORES INERENTES À DIGNIDADE HUMANA... . . . . . . . 3 LUIZ UGEDA • JOÃO SANTA TERRA JR

- 02 •

APLICACIÓN DE TELEDETECCIÓN ESPACIAL PARA LA SOLUCIÓN DE PROBLEMÁTICAS E INJUSTICIAS TERRITORIALES . . . . . . . 27 VÍCTOR HERRERA GONZÁLEZ

- $03 \bullet$

DIREITO AMBIENTAL E CARTOGRAFIA: UM ESTUDO

DE CASO BRASILEIRO SOBRE USO DO SOLO URBANO, RISCOS DE DESASTRES E JUSTIÇA TERRITORIAL . . . . . . . . . 53

JOSÉ RUBENS MORATO LEITE • LARISSA VERRI BORATTI • FERNANDA SALLES CAVEDON-CAPDEVILLE • KLEBER ISAAC SILVA DE SOUZA • KALIU TEIXEIRA • JOSE IRIVALDO ALVES OLIVEIRA SILVA • VALERIANA AUGUSTA BROETTO • MARINA DEMARIA VENÂNCIO • TÔNIA ANDREA DUTRA • EDUARDO BASTOS MOREIRA LIMA • MARIA LEONOR CODONHO • HEIDI MICHALSKI • NATANAEL DANTAS • LUIZ BORGES ROSSETTI BORGES • HUMBERTO FILIPI • LEATRICE FARACO DAROS • ELISA FIORINI BECKHAUSER - EDUARDA MUCCINI • FILIPE BELLINCANTA DE SOUZA • LARISSA BISCHOFF

- $04 \cdot$

EL DERECHO DE ACCESO A LA ENERGÍA EN CUBA. NECESIDAD DEL USO DE UN SIG PARA EL FOMENTO DE ESTE DERECHO.. . 111 YANELYS DELGADO TRIANA • ERNESTO FARIŃAS WONG • JOSÉ GRABIEL LUIS CÓRDOVA

• $05 \cdot$

E A INFRAESTRUTURA DE DADOS ESPACIAIS DO ESTADO DO RIO

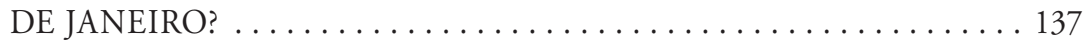
LUIZ UGEDA • JOSÉ AUGUSTO SAPIENZA RAMOS 
PARTE II - JUSTIÇA TERRITORIAL E BACIAS HIDROGRÁFICAS . . 157

- $06 \cdot$

EL SENTIDO E IMPORTANCIA SOCIO-JURÍDICA

DE LAS CUENCAS HIDROGRÁFICAS . . . . . . . . . . . . . . . . . . . 159

JOSÉ IRIVALDO ALVES O. SILVA • ANA L. BURGOS

• $07 \bullet$

CONFLICTOS SOCIO-AMBIENTALES EN BRASIL Y COSTA RICA:

UN ANÁLISIS CRÍTICO DE LOS CASOS DEL PROYECTO

HIDROELÉCTRICO BELOMONTE Y LA MINERÍA EN

LA AMAZONÍA Y DEL PROYECTO HIDROELÉCTRICO

EL DIQUÍS EN EL RÍO GRANDE DE TÉRRABA . . . . . . . . . . . 181

CARLOS E. PERALTA • MARCELA MORENO BUJÁN • JOSÉ IRIVALDO ALVES O. SILVA

- 08 •

MAPEO DE LA INJUSTICIA DEL AGUA EN BRASIL: UNA

HERRAMIENTA FUNDAMENTAL PARA LA TOMA DE

DECISIONES EN EL SUR GLOBAL CON RESPECTO

AL AGUA SUBTERRÁNEA. . . . . . . . . . . . . . . . . . . . . . 219

JOSÉ IRIVALDO ALVES O. SILVA • JOSÉ RUBENS MORATO LEITE

• 09 •

JUSTICIA TERRITORIAL: LA PROTECCIÓN DEL AGUA

MEDIANTE EL USO DE LA INFORMACIÓN GEOGRÁFICA, EL CASO DE LA CUENCA DEL RÍO SANTA LUCÍA. . . . . . . . . . . 241 VIRGINIA FERNÁNDEZ • YURI RESNICHENKO

- $10 \bullet$

APLICAÇÃO DE GEODADOS EM POLÍTICAS PÚBLICAS: CONFLITO ENTRE O INTERESSE PÚBLICO E O PODER ECONÓMICO NA MINERAÇÃO . . . . . . . . . . . . . . . . . . . . . . 259 MONICA FARIA BAPTISTA FARIA 
- $11 \bullet$

ARTICULAÇÃO ENTRE DIREITO À HABITAÇÃO E POLÍTICAS URBANAS (EM ESPECIAL AS POLÍTICAS DE URBANISMO E DE ORDENAMENTO E PLANEAMENTO TERRITORIAL) . . . . . . 267 FERNANDA PAULA OLIVEIRA

- 12 •

PARA ALÉM DOS DESLOCADOS CLIMÁTICOS: OS DESLOCADOS AMBIENTAIS, VÍTIMAS DO PROGRESSO E DE INJUSTIÇAS TERRITORIAIS. . . . . . . . . . . . . . . . 279 ALEXANDRA ARAGÃo

- $13 \cdot$

LOS ÚLTIMOS EPISODIOS DE LA DOCTRINA LÓPEZ OSTRA EN

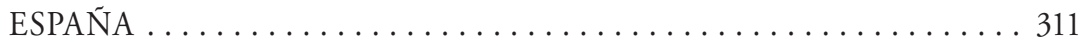
DAVID SAN MARTÍN SEGURA • LUCÍA MUŃOZ BENITO

- $14 \cdot$ JUSTICIA AMBIENTAL EN TIEMPOS DE ECONOMÍA

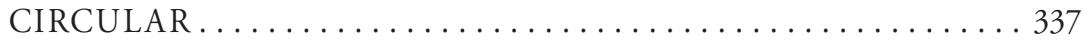
RENÉ JAVIER SANTAMARÍA ARINAS

- 15 •

AS ÁREAS OCUPADAS E OS VAZIOS URBANOS COMO DESAFIOS PARA A PROMOÇÃO DA JUSTIÇA TERRITORIAL NAS CIDADES BRASILEIRAS: O CASO DA CIDADE DE PASSO FUNDO.. . . . . . . 375 CARLA PORTAL VASCONCELLOS

- $16 \cdot$ OS DIREITOS HUMANOS COMO ESTRATÉGIA DE LITIGÂNCIA CLIMÁTICA PARA O ALCANCE DA JUSTIÇA TERRITORIAL NA ERA DO ANTROPOCENO . . . . . . . . . . . . . . . . . 391 ELISA FIORINI BECKHAUSER

PARTE IV - CONCEITOS ESTRUTURANTES.............401 
- $17 \bullet$

ANTROPOCÉNICO. UMA CONCEPÇÃO ANTROPOCENTRISTA

DA DINÂMICA DE MUDANÇA NA MUDANÇA DA DINÂMICA

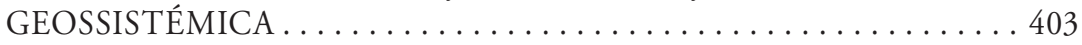

JOSÉ GOMES DOS SANTOS

- $18 \cdot$

LA EFICACIA DE LAS INSTITUCIONES AMBIENTALES EN EL

ANTROPOCENO . . . . . . . . . . . . . . . . . . . . . . 421

NICOLAS J. LUCAS

- $19 \bullet$

DERECHO AL AMBIENTE. DERECHOS HUMANOS. INJUSTICIAS

TERRIORIALES. EVOLUCIÓN DE LA JURISPRUDENCIA

AMBIENTAL INTERNACIONAL . . . . . . . . . . . . 461

SILVIA NONNA Y DELFINA VILA MORET

- $20 \bullet$

DERECHO FUNDAMENTAL A LA BIODIVERSIDAD Y

SERVICIOS DE LOS ECOSISTEMAS . . . . . . . . . . . . . . . . . . . . . 499

LEILA DEVIA

- $21 \cdot$

EL DERECHO AL MEDIO AMBIENTE SANO EN LA CONSTITUCIÓN DE LA REPÚBLICA DE CUBA DE 2019, BAJO EL PRISMA

DE LA REGULACIÓN JURÍDICO-AMBIENTAL. . . . . . . . . . . . . . 529

YANELYS DELGADO TRIANA • GUSTAVO MANUEL HERNÁNDEZ ARTEAGA

- 22 •

EL PATRIMONIO FORESTAL Y LA GESTIÓN DE LOS BOSQUES

ANTE EL NOVÍSIMO CONSTITUCIONALISMO AMBIENTAL

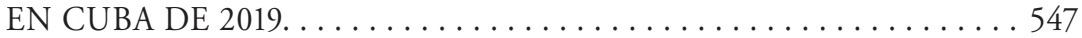

GUSTAVO MANUEL HERNÁNDEZ ARTEAGA

- $23 \cdot$

O ESTATUTO DA CIDADE COMO INSTRUMENTO PARA A PROMOÇÃO DA JUSTIÇA TERRITORIAL NO BRASIL . . . . . . . . . 557 JOÁO TELMO DE OLIVEIRA FILHO

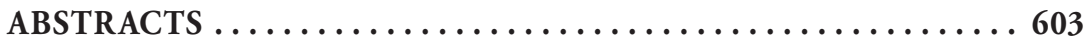




\section{PARTE IV}

\section{CONCEITOS ESTRUTURANTES}


402 


\title{
- 23 - O ESTATUTO DA CIDADE COMO INSTRUMENTO PARA A PROMOÇÃO DA JUSTIÇA TERRITORIAL NO BRASIL
}

\author{
JOÃO TELMO DE OLIVEIRA FILHO ${ }^{1}$
}

\begin{abstract}
Resumo: O presente artigo busca apresentar, de forma resumida, algumas das normas para o desenvolvimento urbano e a promoção da justiça territorial no Brasil, especialmente os dispositivos do Estatuto da Cidade (Lei 10.257/01) e discutir o dilema da efetivação destas normas. Serão apresentados os principais instrumentos de desenvolvimento urbanístico presentes no Estatuto, dividindo-os em instrumentos fiscais e tributários, como o parcelamento e edificação compulsórios e o Imposto sobre a propriedade territorial urbana progressivo no tempo, os institutos jurídicos e políticos de induçáo ao desenvolvimento urbano, como a outorga onerosa e a transferência do direito de construir e os instrumentos de regularização fundiária, que inclui instrumentos previstos pelo Programa Minha Casa, Minha Vida para, ao final, realizar uma breve avaliação da das políticas de desenvolvimento urbanístico no Brasil e discorrer se os instrumentos presentes Estatuto da Cidade sáo capazes de promover a justiça territorial.
\end{abstract}

Palavras-chave: Estatuto da Cidade. Justiça Territorial. IPTU progressivo. Regularização Fundiária. Programa Minha Casa, Minha Vida. Brasil.

Este trabalho busca apresentar algumas das principais diretrizes e normas de planejamento e gestáo urbanos conce-

1 Advogado, doutor em planejamento urbano e regional pela Universidade Federal do Rio Grande do Sul, realizou estágio de pós-doutoramento junto ao CEDOUA - Faculdade de Direito da Universidade de Coimbra, Portugal, como bolsista da CAPES - Governo Brasileiro, processo n. 3116/12-3. Professor da Universidade Federal de Santa Maria - UFSM, Brazil. Email: joaotelmofilho@gmail.com. 
bidos a partir da Constituição Federal de 1988 e presentes no Estatuto da Cidade (Lei 10.257/01) e normas correlatas, em relação à instrumentalização e materialização das políticas de desenvolvimento urbanístico e a promoção da justiça territorial no Brasil. Dentre os instrumentos de indução do cumprimento da função social da propriedade previstos no Capitulo da Política Urbana da Constituição Federal de 1988 e regulamentado no Estatuto da Cidade está o parcelamento, edificação ou utilização compulsórios - PEUC e o imposto sobre a propriedade predial e territorial urbana progressivo no tempo - IPTU progressivo.

Os municípios, mediante lei específica prevista no plano diretor, devem implementar estes instrumentos como forma de conter os vazios urbanos, os imóveis subutilizados e as áreas irregulares que em geral constituem "reservas especulativas, buscando apropriar-se dos investimentos coletivos para valorização dos imóveis" (Brasil, 2015).

A Constituição Federal de 1988 e o Estatuto da Cidade estabelecem que princípio da função social da propriedade urbana assegura o direito a propriedade imobiliária urbana, desde que cumprida a sua função social. Compete ao município, através de lei municipal, promover o controle do processo de desenvolvimento urbano através da política de ordenação do seu território, dentro das diretrizes da sua lei orgânica municipal e seu plano diretor.

Os instrumentos previstos no Estatuto da Cidade como do parcelamento ou edificação compulsória, o imposto sobre a propriedade predial e territorial urbana progressivo no tempo, a desapropriação para fins de reforma urbana, são instrumentos de aplicação deste princípio.

O parcelamento, edificação ou utilização compulsório (PEUC) instrumento que pode ser utilizados pelo Poder Público municipal, como forma de obrigar os proprietários de imóveis urbanos a utilizar socialmente estes imóveis, de acordo com o disciplinado obrigatoriamente no Plano Diretor. Pode ser através do parcelamento de uma área urbana não utilizada ou subutilizada ou a edificação de uma área urbana náo edificada.

Por meio do parcelamento ou edificação compulsória o 
Poder Público municipal condiciona o proprietário a assegurar o uso social da propriedade a um comportamento positivo, de utilizar, construir. Em caso de descumprimento das condiçóes e dos prazos previstos deve aplicar o imposto com a majoração da alíquota pelo prazo de cinco anos consecutivos. No caso de não ser atendida nos cinco anos a obrigação, o Município poderá desapropriar o imóvel para fins de reforma urbana.

O imposto predial e territorial urbano progressivo no tempo tem natureza extrafiscal, caracteriza-se como sanção ao proprietário que não destinou sua propriedade a uma função social. A finalidade do município na utilização do IPTU progressivo no tempo não é a arrecadação, mas o de forçar o proprietário a cumprir com as obrigaçóes previstas no plano diretor, de parcelar ou edificar ampliando o acesso a terra urbanizada ou edificada. Defendido como uma das soluçóes para o problema da especulação imobiliária urbana, o IPTU progressivo ainda não foi regulamentado em muitos municípios brasileiros ou quando regulamentados, aplicados limitadamente ${ }^{2}$.

Outros instrumentos presentes no Estatuto da Cidade de indução ao desenvolvimento urbano são o direito de superfície (art. 21-24), ao direito de preempçáo (art. 25), a outorga onerosa e a transferência do direito de construir (arts. 28 e segs), as operaçóes urbanas consorciadas (arts. 32 a 34) e o consórcio imobiliário (art. 46). São instrumentos que possibilitam novos recursos para as prefeituras municipais investirem em áreas sociais através da promoção de mais-valias urbanas decorrente do "comércio" de índices construtivos e da compensação pelo ônus das urbanizaçóes.

O Estatuto da Cidade também descreve uma série de

2 O estudo levou em consideração os dados da Pesquisa de Informaçóes Básicas Municipais do Instituto Brasileiro de Geografia e Estatística (IBGE/ MUNIC, 2012) identificou 286 Municípios com população acima de $100 \mathrm{mil}$ habitantes que declararam possuir o Plano Diretor (99\% dos municípios), entretanto apenas 91 municípios afirmaram possuir lei específica para aplicação do PEUC, (32\% dos municípios).

A segunda etapa do estudo buscou verificar a existência da lei do PEUC e IPTU progressivo e apenas 25 Municípios possuíam legislação aprovada e com condição de aplicá-la. 
instrumentos de regularizaçâo fundiária, como a Concessão de Uso Especial para Fins de Moradia individual e coletiva (Regulamentado pela Medida Provisória 2.220, de 04 de setembro de 2001), a Concessão do Direito Real de Uso (CDRU), as Zonas Especiais de Interesse Social (ZEIS), o usucapião coletivo (art. 10), a demarcação urbanistica e a legitimação na posse.

Cumpre ressaltar os enormes desafios e o tamanho do problema das ocupaçóes irregulares e precárias no Brasil. Estes instrumentos sáo fundamentais para o acesso e o direito a posse ou a propriedade para milhóes de brasileiros. $\mathrm{O}$ poder público municipal tem o papel fundamental na implementação destes dispositivos que estão intimamente ligados, mas percebe-se no país ainda certo descompasso entre o que é previsto e disposto na legislação federal e a vontade política das prefeituras municipais, agravado pela diminuiçáo dos recursos federais na área de habitação de interesse social.

Em que medida todos estes instrumentos presentes no Estatuto da Cidade sáo capazes de promover a justiça territorial? A legislação urbanística brasileira demonstram tanto avanços quanto retrocessos no processo de efetivação da justiça territorial. Certamente, muito das mudanças e progressos sociais decorrem da evolução legislativa e das instituiçóes democráticas. Uma ampla gama de assuntos diferenciados é tratado pelo Estatuto da Cidade e pela legislação urbanística brasileira. Estes dispositivos promovem a evolução dos modelos tradicional de planejamento e gestão e do modelo dos planos diretores físico-territoriais em uma transição para os planos estratégicos e participativos. Entretanto, a própria complexidade dos processos propostos e as práticas políticas tradicionais dificultam a implementação dos instrumentos previstos na legislaçáo.

\section{BIBLIOGRAFIA}

Brasil. Estatuto da Cidade - Lei no 10.257 de 10 de julho de 2001.

Diário Oficial da União, Brasília, 11 de junho de 2001.

Brasil. Ministério da Justiça e Cidadania. Secretaria de Assuntos

Legislativos. Projeto Pensando o Direito. Pesquisa: Parcelamento, 
edificação ou utilização compulsórios e IPTU progressivo no tempo: regulamentação e aplicação. Coordenadora da pesquisa: Rosana Denaldi, Universidade Federal do ABC - Santo André, São Paulo. Disponível em http://pensando.mj.gov.br/wp-content/ uploads/2015/11/PoD 56 web1.pdf. Acesso em 12/10/19

Oliveira Filho, João Telmo. O Direito do Urbanismo Constitucional. Elementos para a configuração jurídica da disciplina. Revista Brasileira de Direito. Passo Fundo, Ed. Métodos, 2006.

Souza, Marcelo Lopes de. Mudar a cidade: uma introdução crítica ao planejamento e à gestão urbanos. 2. ed. Rio de Janeiro: Bertrand Brasil, 2003. 
562 